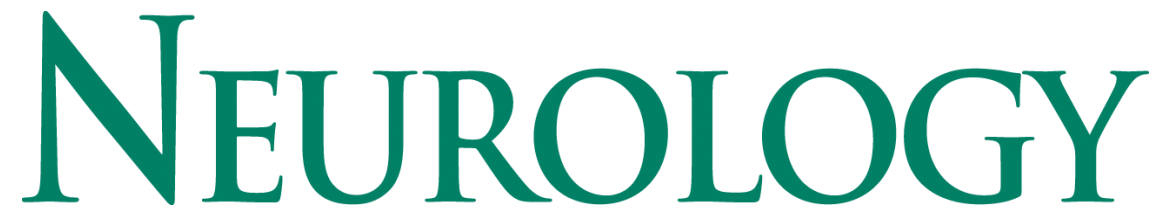

\title{
Differing patterns of temporal atrophy in Alzheimer's disease and semantic
} dementia

C. J. Galton, K. Patterson, K. Graham, M.A. Lambon-Ralph, G. Williams, N. Antoun, B.J. Sahakian and J.R. Hodges

Neurology 2001;57;216-225

This information is current as of March 31, 2008

The online version of this article, along with updated information and services, is located on the World Wide Web at:

http://www.neurology.org/cgi/content/full//57/2/216

Neurology ${ }^{\circledR}$ is the official journal of the American Academy of Neurology. Published continuously since 1951, it is now a weekly with 48 issues per year. Copyright $\left({ }^{\circ} 2001\right.$ by AAN Enterprises, Inc. All rights reserved. Print ISSN: 0028-3878. Online ISSN: 1526-632X.

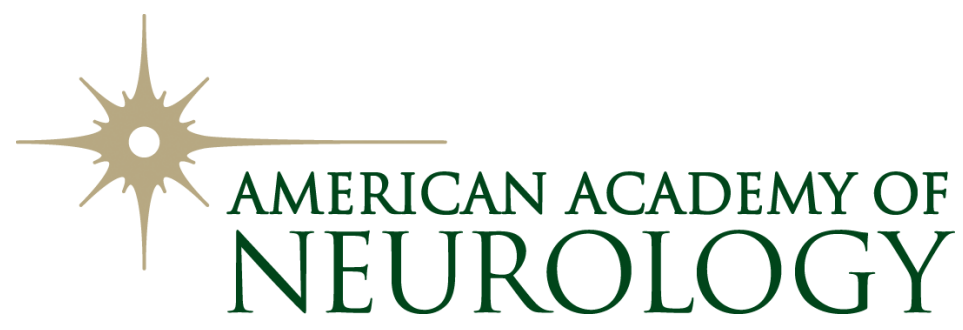




\title{
Differing patterns of temporal atrophy in Alzheimer's disease and semantic dementia
}

\author{
C.J. Galton, MRCP(UK); K. Patterson, PhD; K. Graham, PhD; M.A. Lambon-Ralph, PhD; G. Williams, PhD; \\ N. Antoun, FRCR, FRCP; B.J. Sahakian, PhD; and J.R. Hodges, MD, FRCP
}

Article abstract-Objective: To characterize and quantify the patterns of temporal lobe atrophy in $\mathrm{AD}$ vs semantic dementia and to relate the findings to the cognitive profiles. Medial temporal lobe atrophy is well described in AD. In temporal variant frontotemporal dementia (semantic dementia), clinical studies suggest polar and inferolateral temporal atrophy with hippocampal sparing, but quantification is largely lacking. Methods: A volumetric method for quantifying multiple temporal structures was applied to 26 patients with probable AD, 18 patients with semantic dementia, and 21 matched control subjects. Results: The authors confirmed the expected bilateral hippocampal atrophy in $\mathrm{AD}$ relative to controls, with involvement of the amygdala bilaterally and the right parahippocampal gyrus. Contrary to expectations, patients with semantic dementia had asymmetric hippocampal atrophy, more extensive than AD on the left. As predicted, the semantic dementia group showed more severe involvement of the temporal pole bilaterally and the left amygdala, parahippocampal gyrus (including the entorhinal cortex), fusiform gyrus, and the inferior and middle temporal gyri. Performance on semantic association tasks correlated with the size of the left fusiform gyrus, whereas naming appeared to depend upon a wider left temporal network. Episodic memory measures, with the exception of recognition memory for faces, did not correlate with temporal measures. Conclusions: Hippocampal atrophy is not specific for AD but is also seen in semantic dementia. Distinguishing the patients with semantic dementia was the severe global but asymmetric (left $>$ right) atrophy of the amygdala, temporal pole, and fusiform and inferolateral temporal gyri. These findings have implications for diagnosis and understanding of the cognitive deficits in $\mathrm{AD}$ and semantic dementia.

NEUROLOGY 2001;57:216-225

The topic under investigation in this study is the fate of different temporal lobe structures in two diseases that present with impairment of aspects of memory: $\mathrm{AD}$ and semantic dementia (SD). The earliest and most prominent symptom in $\mathrm{AD}$ is a profound impairment in the ability to acquire and remember new information whether tested by recall or recognition. ${ }^{1-3}$ Semantic memory, the database of conceptual knowledge that gives meaning to sensory experience, is eventually affected in $\mathrm{AD}$ but early in the disease patients show mild and variable impairment of semantic memory. Many patients have purely episodic memory impairment for a number of years. ${ }^{4,5}$ In SD, the presenting feature is a profound breakdown in semantic memory, but in contrast to

Additional material related to this article can be found on the Neurology Web site. Go to www.neurology.org and scroll down the Table of Contents for the July 24 issue to find the title link for this article.
$\mathrm{AD}$, episodic memory is relatively spared in $\mathrm{SD}$, as judged by the patients' preserved orientation, recall of recent autobiographical events, and recognitionbased tests of anterograde memory for pictures. ${ }^{6-8}$

Visual inspection of MRI brain scans has suggested that the hippocampal complex is preserved in $\mathrm{SD}$ compared to $\mathrm{AD}$, and this might explain the patients' normal, or near normal, performance on episodic memory tests. ${ }^{7,9}$ The semantic deficit in SD has been thought to reflect the severe atrophy of polar and inferolateral temporal regions seen on visual inspection. ${ }^{7,9}$ Furthermore, the severity of semantic deficit has been found to correlate with the degree of inferolateral, and not with frontal, atrophy. ${ }^{10}$ The only existent quantitative study of SD involved six patients using an automated voxel-by-voxel based morphometric method, in which individual subjects' brains are compared with a normalized template. While confirming the

\section{$\overline{\text { See also page } 173}$}

From the University Neurology Unit (Drs. Galton and Hodges), Wolfson Brain Imaging Centre (Dr. Williams), and Department of Radiology (Dr. Antoun), Addenbrooke's Hospital, Cambridge; MRC Cognition and Brain Sciences Unit (Drs. Patterson, Graham, and Hodges) and Department of Experimental Psychology (Dr. Lambon-Ralph), University of Bristol; and Department of Psychiatry (Dr. Sahakian), University of Cambridge, Addenbrooke's Hospital, Cambridge, UK.

The research and C.J.G. were funded by a MRC-link project grant to B.J.S., J.R.H., Professor Trevor Robbins, and Dr. James Semple.

Received December 8, 2000. Accepted in final form April 7, 2001.

Address correspondence and reprint requests to Professor John R. Hodges, MRC Cognition and Brain Sciences Unit, 15 Chaucer Road, Cambridge CB2 2EF, UK; e-mail: JRH@mrc-cbu.ac.uk 
severe atrophy of the polar and inferolateral brain regions, this study suggested sparing of the hippocampus and parahippocampal structures. ${ }^{10}$ Atrophy of the medial temporal lobe in $\mathrm{AD}$ has been established using rating scales, ${ }^{11,12}$ measures of hippocampal width and volumes, ${ }^{13-15}$ and measures of entorhinal cortical volume. ${ }^{16}$ Serial MRI coregistration techniques suggest that the atrophy may be more widespread, involving the cortex diffusely even at an early stage of the disease. ${ }^{17}$ It is still unclear whether the medial-temporal atrophy measured in these studies is specific for $\mathrm{AD}$ or can also be a feature of other dementias, because few studies to date have compared AD to other forms of degenerative disease. One notable exception is a pair of studies that compared $\mathrm{AD}$ and frontotemporal dementia (FTD), first using linear measurements ${ }^{18}$ and subsequently volumetric techniques, ${ }^{19}$ and found medial temporal involvement. In both studies, however, patients with progressive aphasia and prominent temporal lobe pathology were excluded; hence, the results, although of interest, do not address the question of primary focus here.

The principal aims of the current study were to characterize and quantify the pattern of atrophy in temporal lobe subregions in patients with SD and mild to moderate stage $\mathrm{AD}$ and to relate these findings to the pattern of neuropsychological deficits found in SD and AD. To achieve this aim we applied a volumetric method derived from other work. ${ }^{13}$ We hypothesized that 1) patients with AD would show hippocampal and possibly parahippocampal gyral atrophy, and 2) the SD group would have polar and inferolateral temporal lobe atrophy, predominantly on the left, with relative sparing of the hippocampus.

Methods. Subjects. A total of 65 subjects participated: 26 with $\mathrm{AD}, 18$ with $\mathrm{SD}$, and 21 controls. The patients all presented to the Memory Disorders Clinic in Addenbrooke's Hospital, Cambridge, over the period 1991 to 1998 and are part of a longitudinal project of cognitive function in the dementias. The study has been approved by the local ethical committee and informed consent was obtained from the patients and relatives. Screening for all patients included a psychiatric interview and medical examination. The diagnosis in both $\mathrm{AD}$ and $\mathrm{SD}$ was made on clinical grounds and not based upon MRI scan findings. The SD group $(\mathrm{n}=18)$ fulfilled local and international consensus criteria for $\mathrm{SD}^{6,20}$ : namely, presentation with a selective impairment in semantic memory causing severe anomia and impaired spoken and written single word comprehension with an impoverished general knowledge about objects and people, with relative sparing of syntax and phonology, normal perceptual skills and nonverbal problem solving, and relatively preserved autobiographic and episodic memory. It should be noted, however, that sparing of episodic memory is relative and as the disease progresses patients fail recall and recognition tests specifically involving verbal material (see discussion). The $\mathrm{AD}$ group consisted of $26 \mathrm{pa}-$ tients with mild to moderate probable $\mathrm{AD}$ who fulfilled National Institute of Neurological and Communicative Disorders and Stroke-Alzheimer's Disease and Related Disorders Association inclusion and exclusion criteria and were chosen from a larger cohort of patients with $\mathrm{AD}$ to match the SD group in terms of disease severity (see below). The demographic and neuropsychological profiles of the two patient groups and the controls are shown in table 1 . The patients with SD were significantly younger than both the $\mathrm{AD}$ and control groups, in keeping with the disease profile of FTD. Although none of the patients in the current study have died, postmortem studies on the Cambridge SD patients have confirmed non-Alzheimer pathology in all cases, in keeping with international experience. ${ }^{21}$ Educational level, as measured by years of education, although lower in the SD group is not significantly different from the other two groups and a linear regression analysis showed no effect of education on the key variables discussed below.

Comparison of disease duration, used as a marker of severity, revealed no significant difference in the duration of symptoms (defined as the time elapsed from the first symptoms, as estimated by patient and spouse, to the MRI scan) between the patient groups, although the onset of disease may not be equally reported in differing dementia syndromes. There was also no difference in the Clinical Dementia Rating $(\mathrm{CDR})^{22}$ between patient groups ( $\mathrm{AD}$ mean $0.9 \pm 0.7$, SD mean $0.9 \pm 0.6$ ), suggest-

Table 1 Demographic data from the subject groups at the approximate time of imaging

\begin{tabular}{|c|c|c|c|c|}
\hline Characteristics & Controls & $\mathrm{AD}$ & Semantic dementia & ANOVA $p$ value \\
\hline Male:female ratio & $12: 9$ & $17: 9$ & $10: 8$ & \\
\hline Mean (SD) age, y & $69.1(7.7)$ & $69.1(7.6)$ & $62.7(7.1)$ & $<0.0005^{\mathrm{a}}$ \\
\hline Mean (SD) duration of symptoms, y & NA & $4.8(3.0)$ & $4.0(2.4)$ & NS \\
\hline Mean (SD) duration of follow-up, $y$ & NA & $3.6(2.3)$ & $3.3(2.4)$ & NS \\
\hline CDR, mean (SD) & NA & $0.9(0.7)$ & $0.9(0.6)$ & NS \\
\hline MMSE, mean (SD) & $29.1(1.0)$ & $22.5(3.2)$ & $21.2(6.9)$ & $<0.0005^{\mathrm{b}}$ \\
\hline
\end{tabular}

a Semantic dementia group younger than the other two groups on Tukey test $(p<0.05)$.

${ }^{\mathrm{b}} \mathrm{AD}$ and semantic dementia groups both worse than control group on Tukey test $(p<0.05)$.

$\mathrm{NA}=$ not applicable; NS = not significant; CDR = Clinical Dementia Rating ${ }^{22} ;$ MMSE $=$ Mini-Mental State Examination. ${ }^{46}$ 
ing the groups are well matched in terms of the functional impact of disease. The clinical diagnosis in our patients has been validated with longitudinal follow-up of at least 2.5 years including regular neuropsychological assessment and observed clinical deterioration. It should be noted that the classification of patient group was independent of the analysis described here. Although none of the patients in this study have come to postmortem yet, 65 patients in our longitudinal project have had pathological examination. Of the 30 patients who had a clinical diagnosis of $\mathrm{AD}$, all had $\mathrm{AD}$ pathology (with or without a mild degree of concomitant vascular or Lewy body pathology). Fifteen patients had a clinical diagnosis of FTD (including five with SD), and all had non-Alzheimer pathology: six had tau-positive Pick bodies, three non-specific gliosis without Pick bodies, two corticobasal ganglionic degeneration, and four ubiquitinpositive inclusions characteristic of motor neuron disease-associated dementia. The subgroup of five patients with SD all had Pick bodies or nonspecific changes.

Control subjects were all healthy volunteers obtained via the subject panel at the MRC Cognition and Brain Sciences Unit. These subjects underwent the same extensive neuropsychological testing as patients and all fell within normal range (see table 1). Subjects with a history of alcoholism, substance abuse, major head injury, or epilepsy or other neurologic disorders were excluded.

Imaging. MRI was performed on a 1.5-T GE Signa MRI scanner (GE Medical Systems, Milwaukee, WI). In all subjects, contiguous MR images of the entire brain were obtained using a volumetric (three-dimensional), inversion recovery prepared, fast gradient echo sequence and the following settings: repetition time $=13.5 \mathrm{~ms}$, echo time $=4.2 \mathrm{~ms}$, inversion time $=650 \mathrm{~ms}$, field of view $=22 \mathrm{~cm}$, slice thickness $=1.5 \mathrm{~mm}$, matrix $256 \times$ 256, 1 excitation. The number of slices was chosen to encompass the whole brain and varied between 116 and 124 for different subjects. Total acquisition time was approximately 9 minutes. Straight coronal images perpendicular to the horizontal plane were obtained. After inspection, the acquired three-dimensional image data set occasionally needed resectioning in order to provide a consistent orientation relative to the brain anatomy across different subjects. This was due to patient movement between the pilot and three-dimensional acquisitions. When needed, these small angular rotations were performed by the Analyze software package (Biomedical Imaging Resource, Mayo Clinic, Rochester, MN). This was necessary for $11 \mathrm{MRI}$ data sets ( one SD).

MRI volumetric measurements. Volumetric analysis was made by a single observer (C.J.G.) who was blind to the subject's details at time of volumetric assessment. The segmentation and data collection took approximately 4 hours per patient. The regions of interest were manually traced on $1.5-\mathrm{mm}$ contiguous coronal slices using Analyze on a Sun Sparcstation 20 (Sun Microsystems, San Francisco, CA). Tracing proceeded from anterior to posterior for all of the temporal subvolumes measured; namely, the anterior temporal pole, hippocampus, amygdala, parahippocampal gyrus, fusiform gyrus, and inferomedial and superior temporal gyri (fig-

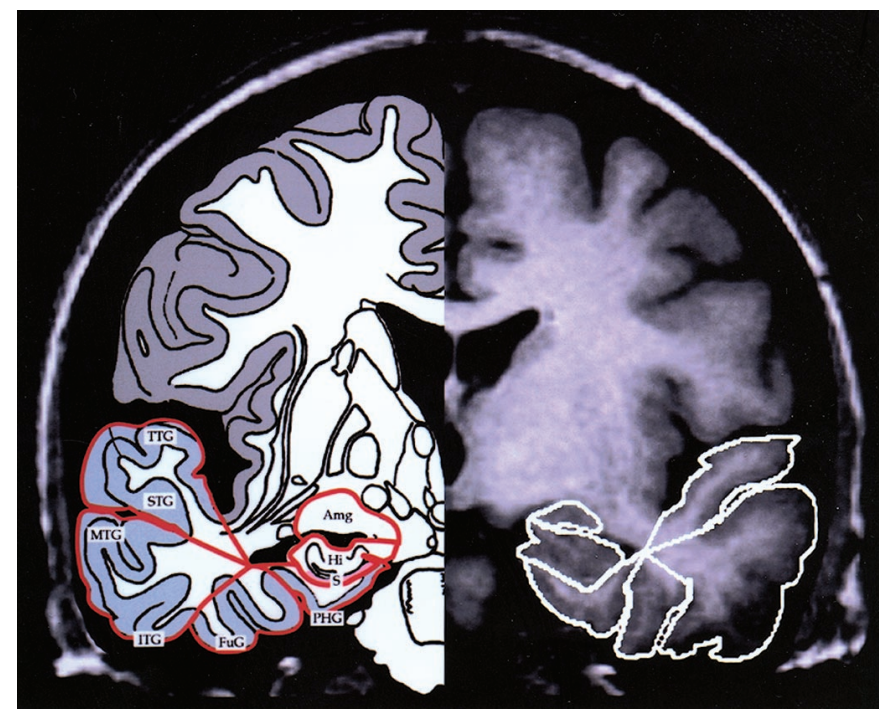

Figure 1. Illustration of the volumetric measures and correspondent anatomy. Amg = amygdala, $\mathrm{Hi}=$ hippocampus, $S=$ subiculum, $P H G=$ parahippocampal gyrus, $F u G=$ fusiform gyrus, ITG = inferior temporal gyrus, $M T G=$ middle temporal gyrus, $S T G=$ superior temporal gyrus, TTG = transverse temporal gyrus. (Adapted from reference 24 .)

ure 1). The intracranial cross-sectional area (in $\mathrm{mm}^{2}$ ) was measured at the slice showing the anterior commissure to correct for head size. In addition, a crosssectional measure of brain atrophy was measured at the level of the splenium on the slice posterior to the hippocampi (figure 2). All anatomic landmarks were defined with reference to hippocampal and brain atlases. ${ }^{23,24}$ The definitions of the temporal subvolumes and their assumed anatomic correlates were as follows.

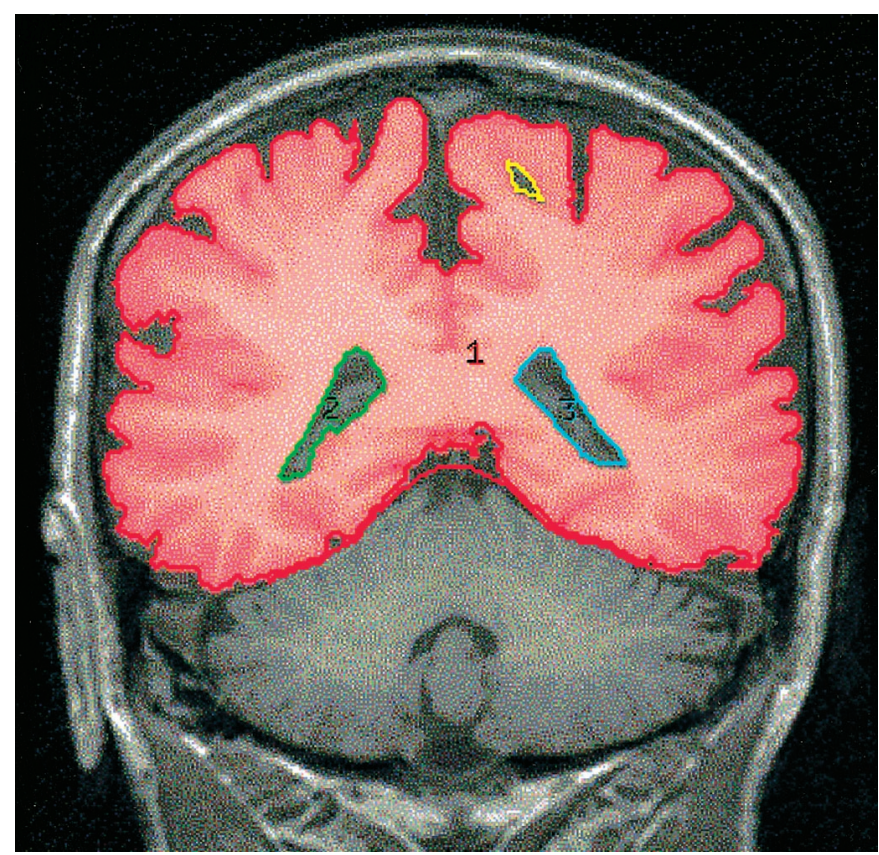

Figure 2. Cross-sectional area of the brain at the level of the splenium. 
Temporal pole-The anterior temporal structures (white and gray matter) were manually traced on all slices where distinguishable until the slice prior to closure of the lateral fissure. This encompassed predominantly temporal pole (Brodmann area [BA] 38) but may include small sections of entorhinal cortex, fusiform, inferior, and middle temporal gyri, and superior temporal gyri (BA 28, 36, 20, 21, and 22).

Amygdala-This structure was defined and segmented according an established technique. ${ }^{25}$ The anterior end of the amygdala was taken as the level of closure of the lateral sulcus to form the entorhinal sulcus. A small section of entorhinal cortex over the medial border was included in the measurement (although entorhinal cortex inferior to the tentorial indentation was excluded).

Hippocampus-The hippocampus (including subiculum) was defined and segmented according to the technique above $^{25}$ but with a few changes. The posterior extent of measurement was the slice on which the sylvian aqueduct (cerebral aqueduct) was visible and the fornix was not included in the posterior slices unless it was embedded in the hippocampal structure. The inferior border was the white matter of the parahippocampal gyrus. This measure is thought to include hippocampal fields CA1 to CA4, dentate gyrus, and subiculum.

The nonhippocampal medial and lateral temporal lobe structures were segmented using a "wheel and spoke" $\operatorname{method}^{13}$ (see figure 1). The reference point for the seg- mentation was the most lateral point of the temporal horn on each slice. This technique was applied to all slices starting with the first slice on which the temporal horn of the lateral ventricle was visible, and ending at the same end slice for hippocampal measurements.

Parahippocampal gyrus-The anatomic correlates of this measure are thought to include most of the entorhinal cortex (BA 28) and some of the perirhinal cortex in the wall of the collateral sulcus (BA 35).

Fusiform gyrus-The neuroanatomic correlates of this measure were thought to be BA 36 and a small part of BA 35 .

Inferior and middle temporal gyri-Owing to variability across subjects these areas were taken together. The medial border was the line from the reference point to the inferior temporal sulcus and the superolateral border was the inferomedial border of the superior temporal gyrus measure. The assumed anatomic correlates for this measure were BA 20 and 21.

Superior temporal gyrus-The anatomic correlates of this measure are thought to be the superior temporal gyrus and transverse temporal gyrus, BA 22 with some 41 and 42 .

The inferred anatomic correlates of our measures are illustrated in figure 1 . The validation of this method was assessed with intrarater reliability measures by repeated measurements on five blinded patient scans. The coefficient of reliability, ${ }^{26}$ a measure of the observed disagreement/chance expected disagreement, was 0.99 for the

Table 2 Neuropsychological data from the subject groups at the approximate time of imaging

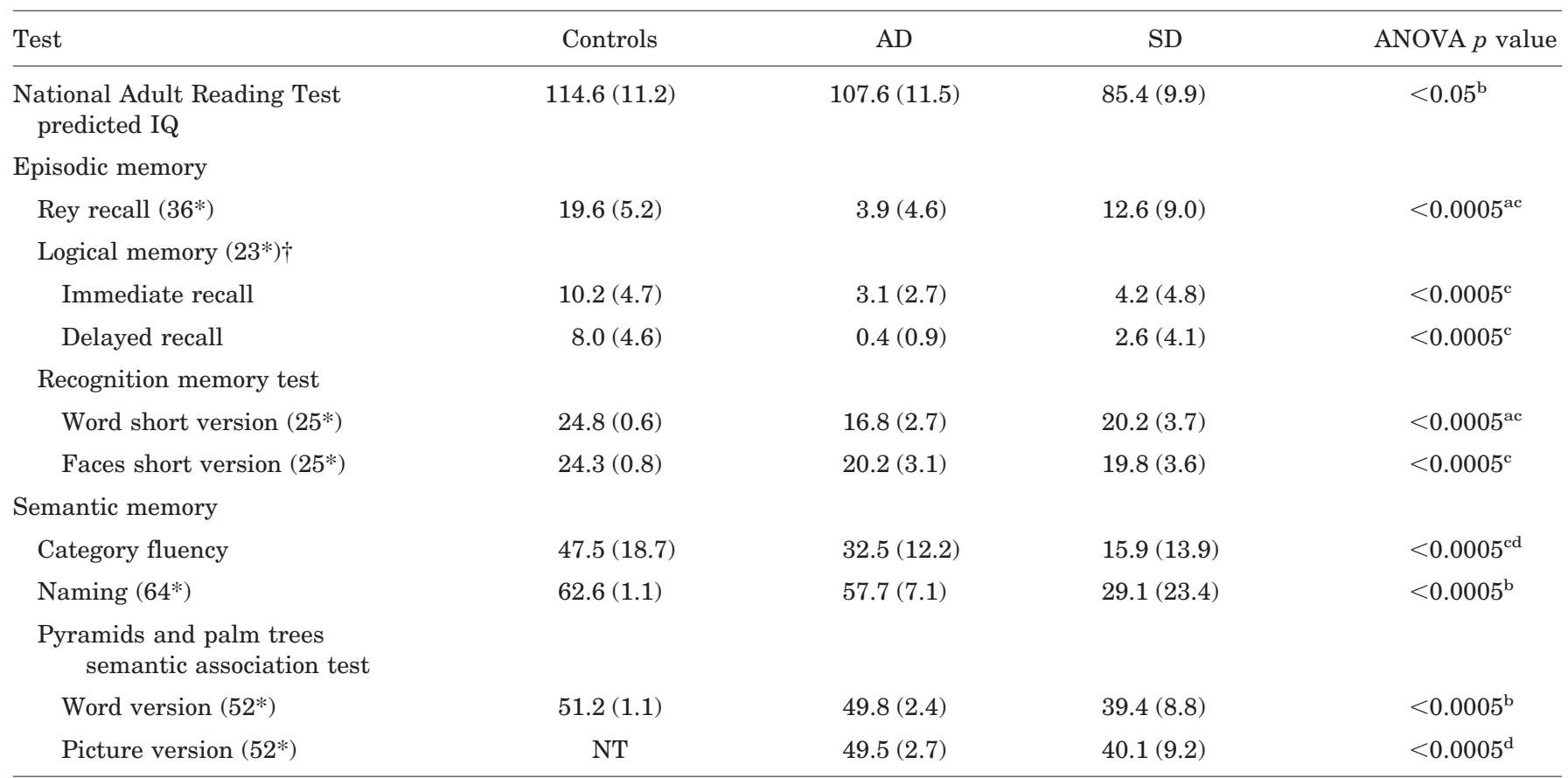

Values are mean $( \pm \mathrm{SD})$.

* Maximum scores are in parenthesis.

$\dagger$ Logical memory (immediate and delayed story recall from the Wechsler Memory Scale-Revised). ${ }^{29}$

${ }^{a} \mathrm{AD}$ group worse than other two groups on Tukey test $(p<0.05)$.

${ }^{\mathrm{b}}$ Semantic dementia (SD) group worse than other two groups on Tukey test $(p<0.05)$.

${ }^{\mathrm{c}} \mathrm{AD}$ and SD groups both worse than control group on Tukey test $(p<0.05)$.

${ }^{\mathrm{d}} \mathrm{SD}$ group worse than $\mathrm{AD}$ group on Tukey test $(p<0.05)$.

$\mathrm{NT}=$ not tested 
Table 3 Results of volumetric analysis of each brain region by group (volumes normalized and expressed as $\mathrm{mm}$ )

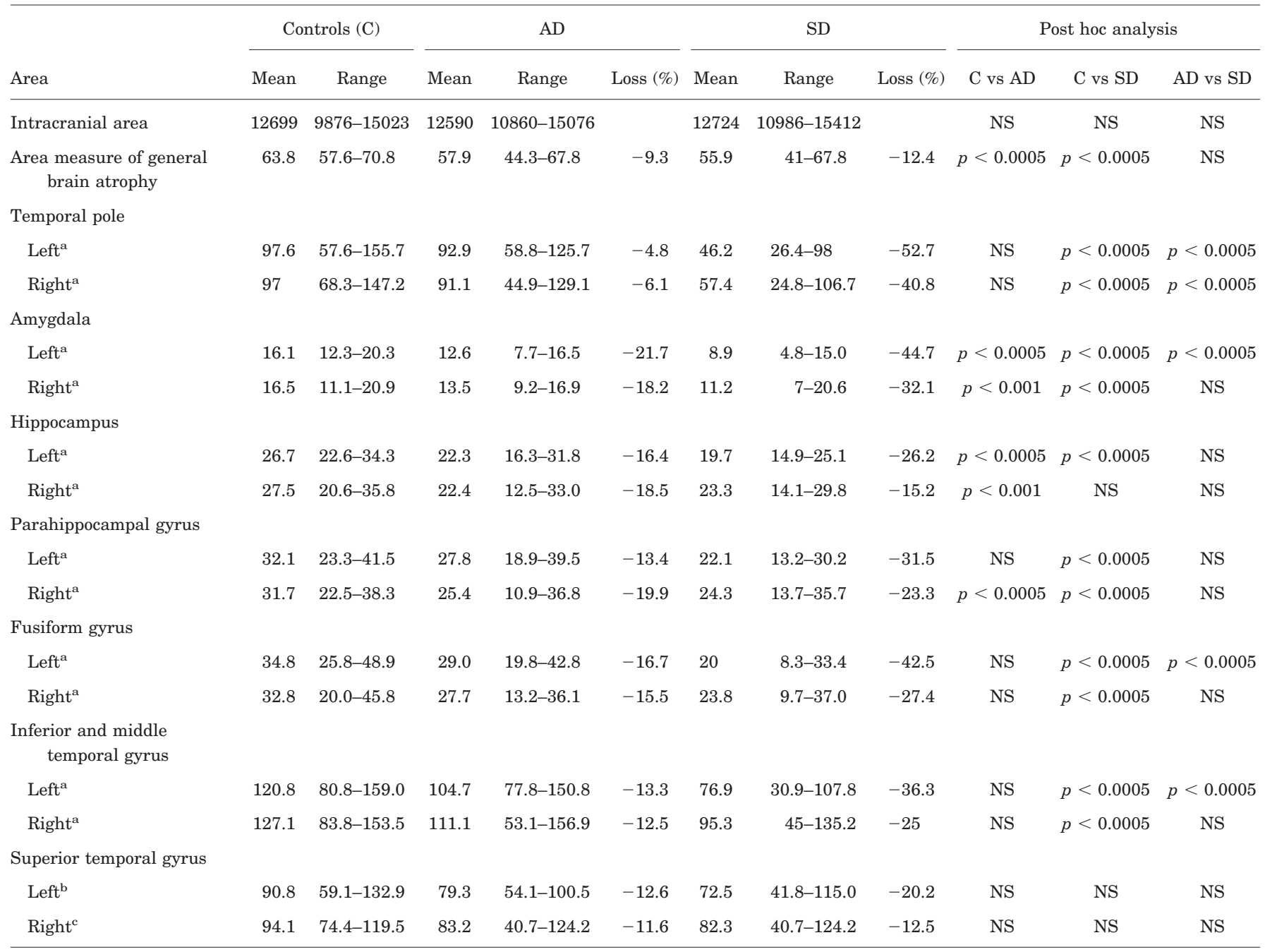

${ }^{\text {a }}$ Main effect $p<0.0005$.

${ }^{\mathrm{b}}$ Main effect $p=0.002$.

${ }^{\mathrm{c}}$ Main effect $p=0.04$.

$\mathrm{SD}=$ semantic dementia; $\mathrm{NS}=$ not significant.

anterior temporal pole, 0.85 for the amygdala, 0.95 for the hippocampus, 0.91 for the parahippocampal gyrus, 0.99 for the fusiform gyrus, 0.96 for the inferior and middle temporal gyri, and 0.96 for the superior temporal gyri. The coefficient of variation of these measures was under $6 \%$ in all cases except for the amygdala, which was $9 \%$ (for supplementary data, please access www.neurology.org and access the title link for this article). The online version of this article, at www.neurology.org, also includes scan-rescan validation, where five control subjects were scanned twice within a few weeks; the rater was blind to name and date of scan. The volumetric data were normalized using the cross-sectional area measure ${ }^{27}$ as (volume/intracranial area) $\times 100$. Volume loss was defined as percent difference from the control mean.

Statistical analysis. The data were analyzed using SPSS 10.0 for Windows (Chicago, IL). Demographic and neuropsychological variables were compared using analysis of variance (ANOVA) with post hoc Tukey HSD tests when appropriate. The mean scores on the rating scales and the volumetric analysis were also assessed using
ANOVA and Tukey post hoc comparisons. The effects of age on the volumetric results were not significant when analyzed using linear regression; therefore, unadjusted means were compared. The post hoc significance tests were adjusted for multiple comparisons using the Bonferroni correction such that the significant value for post hoc tests in table 2 was $<0.001$ and for the post hoc tests shown in the online version of this article (www.neurology.org) was $<0.0009$.

Results. Neuropsychological measures. The results, shown in table 2, confirm the differing cognitive profiles between groups. There was a significant difference in National Adult Reading Test scores with the SD group scoring below the control and $\mathrm{AD}$ groups, which reflects the surface dyslexia typically observed in SD. ${ }^{6}$ As predicted, the AD group had significantly lower scores than the other two groups on most tests of episodic memory including Recognition Memory for words ${ }^{28}$ and recall of the Rey figure, as well the copy of the Rey figure, a 


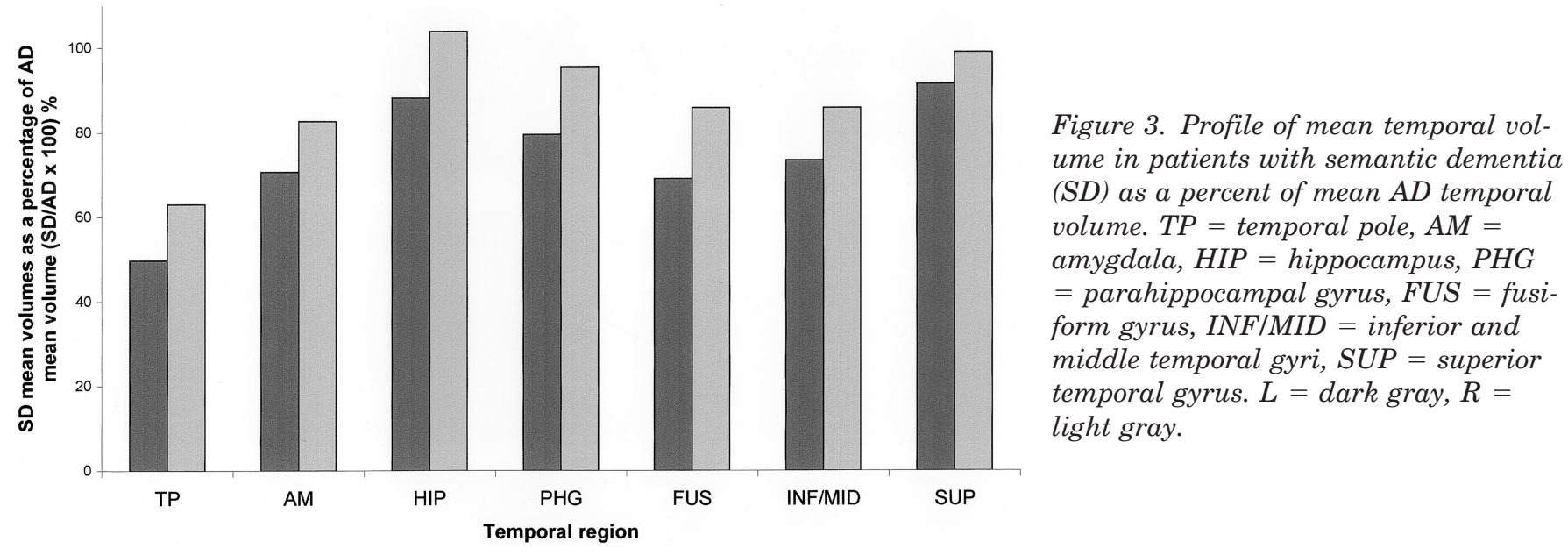

visuospatial task. The patients with SD were impaired on the episodic tasks compared to controls but not as severely impaired as the $\mathrm{AD}$ group, except on recognition memory for faces and logical memory (immediate and delayed recall ${ }^{29}$ ), which was equivalent in $\mathrm{AD}$ and SD. By contrast, on tests of semantic memory, although the mean scores of the $\mathrm{AD}$ group were a little lower than the controls, their performance was within normal limits on all of these assessments except for category fluency. The SD group, on the other hand, showed the expected profound deficits on both verbal and visually based semantic tasks (category fluency, semantic battery naming, ${ }^{2}$ and the Pyramids and Palm trees test of associative semantic knowledge for pictures and words ${ }^{30}$ ). Performance on visuoperceptual and visuospatial tasks, as tested by the Visual Object and Space Perception Battery, ${ }^{31}$ was unimpaired in all groups.

Volumetric results. The mean corrected volumes of the various regions together with the percentage loss compared to the control mean are shown in table 3 . In figure 3 the differences between the $\mathrm{AD}$ and $\mathrm{SD}$ groups are highlighted. A series of ANOVA revealed highly significant main group effects for the temporal pole, amygdala, hippocampus, parahippocampus, fusiform gyrus, and inferior and middle temporal gyri, all bilaterally $(p<0.005)$, and both superior temporal gyri $(p<0.05)$. Post hoc analyses confirmed that relative to controls, the $\mathrm{AD}$ group had significant atrophy of the amygdala and hippocampus bilaterally, and right sided atrophy of the parahippocampal gyrus. By contrast, the SD group showed significant atrophy, relative to controls, for all regions except the right hippocampus and the superior temporal gyri. Comparison of the two patient groups showed greater atrophy in $\mathrm{SD}$ than $\mathrm{AD}$ of the temporal pole bilaterally and, on the left, the amygdala, hippocampus, and the parahippocampal, fusiform, and inferior and middle temporal gyri. The distribution of the temporal pole and hippocampal volumes for each subject are shown in figure 4, $\mathrm{A}$ and $\mathrm{B}$ : the $\mathrm{AD}$ and $\mathrm{SD}$ groups can be distinguished on the basis of the temporal pole volumes, but there was considerable overlap in hippocampal volumes among the three groups. We confirmed this impression by performing a discriminant analysis to compare the ability of the temporal pole and hippocampal volumes to discriminate between groups. Comparing between groups, the temporal pole volumes were able to correctly classify $91 \%$ of the $\mathrm{AD}$ and SD groups, $92 \%$ of the SD and control groups, and could not distinguish between the $\mathrm{AD}$ and control groups. The left hippocampal volume correctly distinguished $59 \%$ of the $\mathrm{AD}$ and SD groups, $85 \%$ of the SD and control groups, and $75 \%$ of $\mathrm{AD}$ and control groups. It is notable that in the $\mathrm{AD}$ group an equivalent proportion of cases showed left and right hippocampal atrophy, but for SD a far greater proportion have small left-sided hippocampal volumes.

Results by severity. To examine the effects of the stage of disease, as measured by severity, the $\mathrm{AD}$ and SD groups were divided into mild and moderate subsets on the basis of a cut-off Mini-Mental State Examination (MMSE) score of 22. We predicted early involvement of the temporal pole in SD, with preservation of medial temporal structures until later in the disease. Likewise, one might expect the milder $\mathrm{AD}$ subgroup to have atrophy restricted to the hippocampus and parahippocampal gyrus, with spread to other temporal areas only in the moderate group. The comparisons of the mild and moderate $\mathrm{AD}$ and $\mathrm{SD}$ groups with the control group are shown in the online version of this article (www.neurology.org). In keeping with our prediction, even patients in the mild SD subgroup were found to have bilateral temporal polar and amygdalar atrophy bilaterally, plus leftsided atrophy of the hippocampus, parahippocampal, fusiform, and inferior and middle temporal gyri. In the moderate SD group, the atrophy also involved the right parahippocampal, fusiform, and inferior and middle temporal gyri. It is notable that the right hippocampal volume in both the early and late stage SD groups was no different from controls, although there was a trend in the later group $(p=0.006)$. In the mild $\mathrm{AD}$ subgroup, no region differed from the control group, although there was a trend in the left amygdala $(p=0.003)$. In the more demented $\mathrm{AD}$ group atrophy of the left amygdala and right parahippocampus reached significance although there was a trend for both hippocampi $(p<$ 0.005).

Correlation with neuropsychology. The relationship of the temporal subregions to neuropsychological measures was analyzed using partial correlations adjusting for general brain atrophy. We explored the relationship between performance on semantic tasks and target brain 


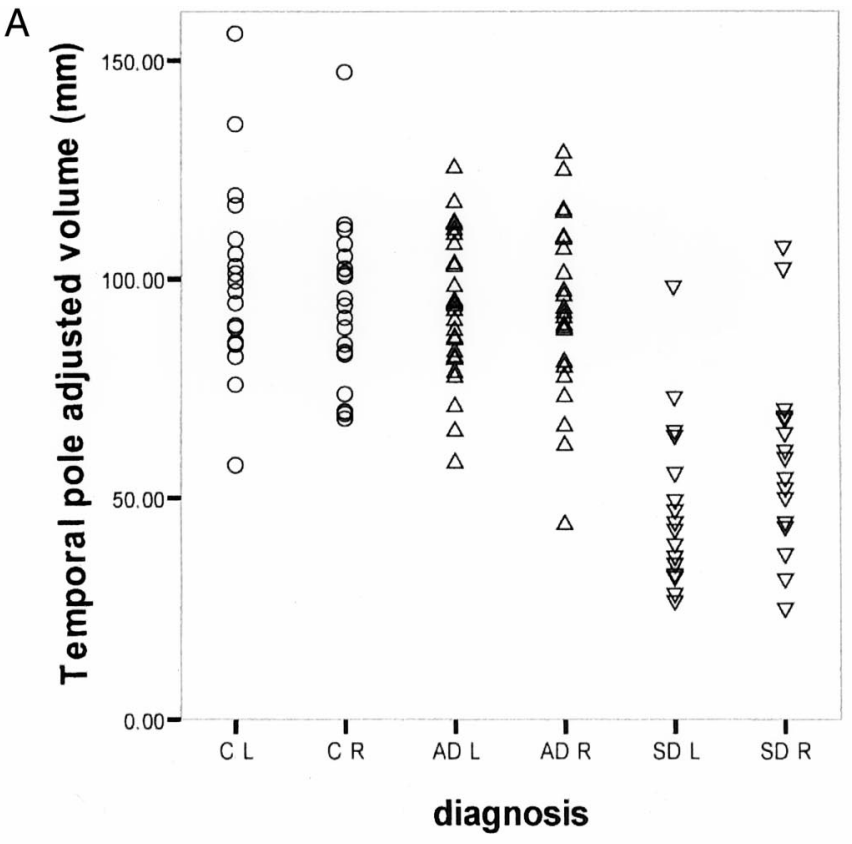

B

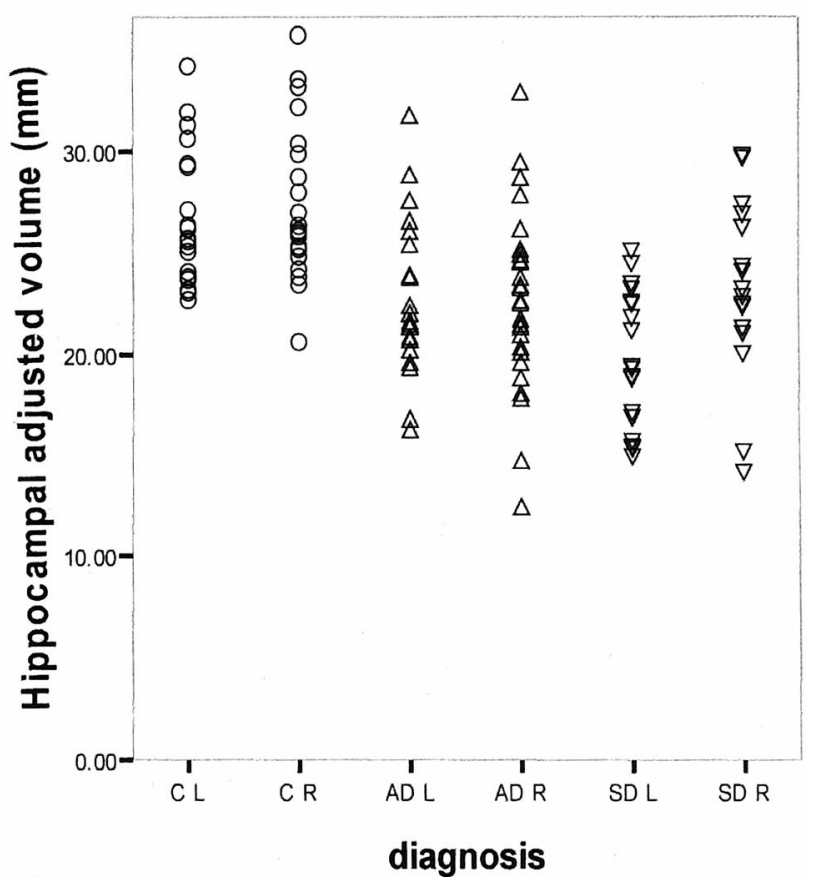

Figure 4. (A) Scatterplot of temporal pole volumes $\left(\mathrm{mm}^{2}\right)$ by group. (B) Scatterplot of hippocampal volume $\left(\mathrm{mm}^{2}\right)$ by group. $C L=$ control left side, $C R=$ control right side, $A D L=A D$ left side, $A D R=A D$ right side, $S D L=s e$ mantic dementia left side, $S D R=$ semantic dementia right side.

regions in a combined group of $\mathrm{AD}$ and $\mathrm{SD}$ patients with the assumption that the neural basis of semantic memory impairment is equivalent in the two clinicopathologic syndromes, both of which affect the temporal neocortex. Because the cause of the episodic memory impairment is likely to be multifactorial, the $\mathrm{AD}$ and $\mathrm{SD}$ groups were analyzed separately. After correction for multiple comparisons, the volume of the left fusiform correlated with all the semantic measures $(r=0.57$ to $0.67, p<0.0005$ ); additionally, the left temporal pole and inferior and middle temporal gyri correlate with the naming tests (semantic naming and category fluency) $(r=$ 0.57 to $0.63, p<0.0005$ ). The correlation matrix is shown in the online version of this article (www.neurology. org). There was no significant relationship between medial temporal lobe structures and semantic memory tasks. Surprisingly, there was very little correlation between episodic recall tasks and any anatomic region. The only significant correlation, after Bonferroni correction, was between the size of the right parahippocampal gyrus and the recognition memory test for faces in the $\mathrm{AD}$ group $(r=0.69, p<$ 0.0005). The correlations are shown in the online version of this article (www.neurology.org). Potential reasons for this result are debated in the discussion.

Discussion. Our study provides systematic assessment and quantification of the pattern of temporal lobe atrophy on MRI in SD and compares SD with AD. Contrary to prior reports based upon clinical assessment of MRI scans, ${ }^{6,7}$ we have demonstrated significant hippocampal atrophy in SD, which differed from than that seen in $A D$ in one respect: whereas in $\mathrm{AD}$ both hippocampi were involved symmetrically, in SD there was an asymmetric pattern. In addition, the patients with SD showed more extensive involvement of the temporal pole bilaterally as well as the left amygdala and parahippocampal, fusiform, and inferior and middle temporal gyri than did patients with AD. The SD group was distinguishable from the AD group by the gross atrophy of the polar and left inferolateral temporal areas.

Our study confirms the well-established finding of significant hippocampal atrophy in $\mathrm{AD}$ compared to controls ${ }^{13,15}$ and highlights the lack of clear differentiation between age-matched controls and patients with relatively early stage disease: the mean percentage of tissue loss was 16 to $19 \%$ with considerable overlap between controls and patients.

The most surprising finding of the study was the demonstration that the SD group had hippocampal atrophy. The mean hippocampal size was actually smaller on the left in the SD group than in the patients with $\mathrm{AD}$. Hippocampal atrophy, therefore, is not disease or syndrome specific. Although the hippocampus has not previously been measured volumetrically in SD, a recent study of six cases using a voxel-based morphometric method suggested sparing of this structure in the context of global neocortical atrophy. ${ }^{10}$ The failure to identify hippocampal abnormality using the morphometric technique possibly reflects the limited resolution of the voxel-by-voxel method for small complex structures such as the hippocampus as the technique relies on the automated comparison of individual subjects with a normalized template derived from the amalgamation of a number of normal control MRI.

As mentioned previously, there is accumulating evidence that the entorhinal cortex, located in the parahippocampal gyrus, is affected in early AD. ${ }^{16}$ 
Our volumetric method confirmed AD parahippocampal atrophy, which was equivalent in degree (13 to 19\%) to the loss of hippocampal volume. The parahippocampal gyrus was even more severely affected in the $\mathrm{SD}$ than the $\mathrm{AD}$ group, and this reached significance for the left side (mean volume loss left $32 \%$, right $23 \%$ ). In a prior volumetric study of patients with FTD, which excluded those with SD, less severe atrophy of the hippocampus was found compared to their $\mathrm{AD}$ group, but equally severe entorhinal cortical atrophy (27 to $28 \%$ loss) with no laterality effects was present. ${ }^{19}$

The question of which neuroanatomic areas are affected early in the disease process in both $\mathrm{AD}$ and SD is of considerable interest. In this cross-sectional study we attempted to address this question by dividing the patients into subgroups based on MMSE score. On the basis of current neuropathologic evidence $^{32,33}$ we predict that the entorhinal cortex (included in the parahippocampal measure) would show the earliest changes in $\mathrm{AD}$ with later involvement of the hippocampal complex proper. Quantitative neuropathologic data in SD are not available on which to base predictions but the neuropsychological data ${ }^{7-9}$ would predict anterolateral cortical atrophy with hippocampal sparing in the earliest stages. Surprisingly, in the mild $\mathrm{AD}$ group the first temporal area differing from the control group was the left amygdala. In the SD group, by contrast, the earliest changes appear to involve the temporal poles and amygdalae bilaterally together with the left parahippocampal, fusiform, and inferolateral gyri. Progression from mild to severe was characterized by spread of atrophy to the right parahippocampal gyrus and inferolateral cortices. These differing early profiles suggest that distinct neuronal networks are affected in the two diseases.

The remaining temporal lobe structures have been assessed very rarely in $\mathrm{AD}$ and not at all in SD. Our hypothesis that the polar, fusiform, and inferolateral temporal regions would be significantly more atrophied in the SD group, compared to $\mathrm{AD}$, was upheld. Using similar volumetric measures, a $20 \%$ reduction in the fusiform gyrus in patients with $\mathrm{AD}$ has been demonstrated, which was significantly greater than in their controls, but this possibly reflects the more advanced state of their cases (mean MMSE 18.3). ${ }^{13}$

The findings in our $\mathrm{AD}$ group are compatible with the known distribution of neuropathologic changes in $\mathrm{AD}$. The earliest site of neurofibrillary tangle and plaque pathology is thought to be the transentorhinal region with subsequent spread to the hippocampal complex before involvement of the lateral temporoparietal multimodal association cortex. ${ }^{32,33}$ Most of our patients were at the stage of showing clear-cut amnesia plus other definite, but often mild, cognitive deficits, such as impaired category fluency or copy of the Rey figure; this pattern suggests that the bulk of pathology was probably confined to the medial temporal lobe. The issue of whether entorhinal cortical involvement is detectable on MRI before hippocampal atrophy, or vice versa, in $A D$ cannot be answered by this current study.

In SD, much less is known about the spread of pathologic change. The fact that the left temporal pole, amygdala, and fusiform gyrus showed the greatest degree of atrophy clearly implies that these structures bear the brunt of the pathology, which is in keeping with postmortem reports. ${ }^{8,21} \mathrm{~A}$ recent meta-analysis identified 13 cases meeting clinical criteria for SD who had postmortem brain examinations. ${ }^{21}$ All cases had non-Alzheimer pathology: some had classic Pick's disease with intraneuronal argyrophilic tau-positive inclusions (Pick bodies), whereas others had identical changes without specific inclusions. Recently, three cases of SD have been reported with ubiquitin-positive tau-negative inclusion bodies. ${ }^{34}$ Most pathological studies agree that the focus of pathology includes the anterior temporal regions, temporal pole and amygdala, and inferomedial temporal cortices with relative sparing of the superior temporal gyrus. ${ }^{8,21}$ There is, however, variability in the involvement of the hippocampus, with some reports of severe involvement ${ }^{34,35}$ and others of relative sparing. ${ }^{36}$

Turning to the cognitive implications of our findings in SD, the two main issues are the critical locus for the genesis of semantic memory breakdown and possible explanations for the sparing of recent episodic memory. Many anecdotal reports suggest that patients with SD are well orientated with good recall of recent personal events, which has now been confirmed empirically. ${ }^{7,9}$ Patients with SD show a reversal of the usual temporal gradient seen in amnesia and in $\mathrm{AD}$, with normal or near normal recent autobiographical memory relative to the more distant past. ${ }^{7,8}$ Performance on standard tests of anterograde episodic memory depends critically upon the type of material, mode of assessment, and stage of disease. Verbal recall is uniformly poor, reflecting the SD patients' anomia. Visually based memory is typically much better and performance on recognition based tests may be normal.7,9

The finding of a virtually equivalent degree of hippocampal atrophy in $\mathrm{AD}$ and $\mathrm{SD}$ raises the question of why patients with even very early $A D$ are so amnesic. We can only speculate that the differing pathology with differing distribution may be of relevance. The bilateral nature of the pathologic process in $\mathrm{AD}$, which first deafferentates the hippocampus by destroying the entorhinal input zone and then spreads into the hippocampus complex proper, may be the critical factor. An additional factor in $\mathrm{AD}$ is the early involvement of the basal forebrain cholinergic system (BFCS), particularly the nucleus basalis. ${ }^{37}$ It may well be that this pathology contributes significantly to the profound episodic memory deficit in AD. The more asymmetric nature of the pathology in SD and its presumed spread from the polar regions posteri- 
orly, plus the sparing of the BFCS, may be key factors that allow the system to compensate in SD. It is interesting in this regard that the recognition memory for pictures and faces (described above) is heavily dependent upon perceptual inputs that are likely to enter the hippocampal complex in the caudal portion and are biased to the right. The rightsided medial temporal structures were more spared in our SD group particularly in the early stages. An alternative explanation is that other cortical areas, important for episodic memory function, are differentially affected in $\mathrm{AD}$ and SD. The current study did not measure brain regions outside the temporal lobe and cannot therefore adjudicate between various possibilities. Functional imaging studies of memory in normal subjects have highlighted the role of the frontal lobes in memory encoding and retrieval. ${ }^{38,39}$ It is possible that differential frontal involvement could account for the differences in memory function between the two groups. One might expect, however, that the SD group would have more severe frontal involvement based on the pathologic and behavioral features of the disease, although pathologic involvement of the frontal cortex is increasingly recognized in $\mathrm{AD} .{ }^{40}$ Other cortical areas that may be differentially affected by $\mathrm{AD}$ and SD pathology are the posterior cingulate and retrosplenial cortex. Resting metabolic FDG-PET studies of patients in the earliest stages of $\mathrm{AD}$ suggest that a common early feature is posterior cingulate hypometabolism. ${ }^{41}$ It is of interest in this context that we found very little correlation between performance on the episodic memory tasks and the medial temporal lobe structures, suggesting that other factors play an important part in genesis of the episodic memory deficits in AD. The only significant association was between recognition memory for faces and the degree of atrophy of the right parahippocampal gyrus, in keeping with the proposed role of nonhippocampal structures in recognition-based memory. ${ }^{42}$

Another factor to consider is that equivalent degrees of atrophy of the hippocampus in different diseases does not imply that there is loss of the same cell population in both disorders. Indeed, pathological differences may underlie the different patterns of atrophy in the mild and severe groups in this study. In $\mathrm{AD}$, there are high densities of amyloid plaques and neurofibrillary tangles with neuronal loss in the transentorhinal and entorhinal cortex, CA1, and all fields of the hippocampus, with increasing severity. ${ }^{32,33}$ In FTD, intraneuronal inclusions are typically present in the granular neurons of the dentate gyrus, ${ }^{34}$ or within the pyramidal cell areas of CA1 and the subiculum, where there is neuronal loss. ${ }^{8}$

Can we draw any conclusions regarding the locus of the semantic deficit in SD? Functional imaging studies of normal participants, using semantic activation paradigms, have implicated a widely distributed network centering on the fusiform gyrus, inferolateral temporal lobe, occipitotemporal junction, and angular gyrus, plus extensive frontal activation, all mainly in the left hemisphere. ${ }^{43,44} \mathrm{In}$ our study, analysis of the relationship between performance on semantic tasks and temporal lobe structures suggested that semantic memory, as tested by both verbal and visual semantic association tasks, appears to depend critically upon the left fusiform gyrus. When a naming component was included in the task (picture naming, category fluency), the left temporal pole and inferolateral temporal regions were also significantly correlated, suggesting that naming involves a more extensive left-sided network. Recently, interest has also focused on a possible role for the perirhinal cortex in semantic memory. ${ }^{45}$ Unfortunately, the site of the perirhinal cortex remains somewhat controversial in man, but is thought to occupy the banks of the collateral sulcus, lateral to the entorhinal cortex (see figure 1), and to extend rostrally on the medial aspect of the temporal pole. Although this region was severely atrophied in the $\mathrm{SD}$ group, there was no correlation between the semantic tasks and the parahippocampal gyrus. ${ }^{46}$

\section{Acknowledgment}

The authors thank Rachel Swainson and Sharon Davies for help with background neuropsychological data collection, Leslie Hurn and Ilse Joubert for MRI support, and Peter Watson for statistical advice.

\section{References}

1. Grady CL, Haxby JV, Horwitz B, et al. Longitudinal study of the early neuropsychological and cerebral metabolic changes in dementia of the Alzheimer's type. J Clin Exp Neuropsychol 1988; 10:576-596.

2. Hodges JR, Patterson K. Is semantic memory consistently impaired early in the course of Alzheimer's disease? Neuroanatomical and diagnostic implications. Neuropsychologia 1995;33:441-459.

3. Welsh K, Butters N, Hughes J, Mohs R, Heyman A. Detection of abnormal memory decline in mild cases of Alzheimer's disease using CERAD neuropsychological measures. Arch Neurol 1991;48:278-281.

4. Perry RJ, Hodges JR. Differentiating frontal and temporal variant frontotemporal dementia from Alzheimer's disease. Neurology 2000;54:2277-2284.

5. Perry RJ, Watson P, Hodges JR. The nature and staging of attention dysfunction in early (minimal and mild) Alzheimer's disease: relationship to episodic and semantic memory impairment. Neuropsychologia 2000;38:252-271.

6. Hodges JR, Patterson K, Oxbury S, Funnell E. Semantic dementia: progressive fluent aphasia with temporal lobe atrophy. Brain 1992;115:1783-1806.

7. Graham KS, Hodges JR. Differentiating the roles of the hippocampal complex and the neocortex in long-term memory storage: evidence from the study of semantic dementia and Alzheimer's disease. Neuropsychology 1997;11:77-89.

8. Snowden JS, Neary D, Mann DMA. Fronto-temporal lobar degeneration: fronto-temporal dementia, progressive aphasia, semantic dementia. Clinical neurology and neurosurgery monographs. New York: Churchill Livingstone, 1996.

9. Graham KS, Simons JS, Pratt KH, Patterson K, Hodges JR. Insights from semantic dementia on the relationship between episodic memory and semantic memory. Neuropsychologia 2000;38:313-324.

10. Mummery CJ, Patterson K, Price CJ, Frackowiak RSJ, Hodges JR. A voxel based morphometry study of semantic 
dementia: the relation of temporal lobe atrophy to cognitive deficit. Ann Neurol 2000;47:36-45.

11. Scheltens P, Leys D, Barkhof F, et al. Atrophy of medial temporal lobes on MRI in "probable" Alzheimer's disease and normal aging: diagnostic value and neuropsychological correlates. J Neurol Neurosurg Psychiatry 1992;55:967-972.

12. DeLeon M, George A, Golomb J, et al. Frequency of hippocampal formation atrophy in normal aging and Alzheimer's disease. Neurobiol Aging 1997;18:1-11.

13. Convit A, DeLeon M, Tarshish C, et al. Specific hippocampal volume reductions in individuals at risk for Alzheimer's disease. Neurobiol Aging 1997;18:131-138.

14. Jack CR, Petersen RC, O'Brien PC, Tangalos EG. MR-based hippocampal volumetry in the diagnosis of Alzheimer's disease. Neurology 1992;42:183-188.

15. Killiany R, Moss MB, Albert MS, Sandor T, Tieman J, Jolesz F. Temporal lobe regions on magnetic resonance imaging identify patients with early Alzheimer's disease. Arch Neurol 1993;50:949-954.

16. Juottonen K, Laakso M, Insausti R, et al. Volumes of the entorhinal and perirhinal cortices in Alzheimer's disease. Neurobiol Aging 1998;19:15-22.

17. Fox N, Warrington E, Seiffer A, Agnew S, Rossor M. Presymptomatic cognitive deficits in individuals at risk of familial Alzheimer's disease. Brain 1998;121:1631-1639.

18. Frisoni GB, Beltramello A, Geroldi C, Weiss C, Bianchetti A, Trabucchi M. Brain atrophy in frontotemporal dementia. J Neurol Neurosurg Psychiatry 1996;61:157-165.

19. Frisoni GB, Laakso MP, Beltramello A, et al. Hippocampal and entorhinal cortex atrophy in frontotemporal dementia and Alzheimer's disease. Neurology 1999;52:91-100.

20. Neary D, Snowden JS, Gustafson L, et al. Frontotemporal lobar degeneration: a consensus on clinical diagnostic criteria. Neurology 1998;51:1546-1554.

21. Hodges JR, Garrard P, Patterson K. Semantic dementia. In: Kertesz A, Munoz DG, eds. Pick's disease and Pick's complex. San Diego: Academic Press, 1998;83-102.

22. Hughes CP, Berg L, Danziger WL, Cohen LA, Martin RL. A new clinical scale for the staging of dementia. Br J Psychiatry 1982;140:566-572.

23. Duvernoy HM. The human hippocampus: functional anatomy, vascularization and serial sections with MRI. Berlin: Springer-Verlag, 1998.

24. Mai JK, Assheuer J, Paxinos G. Atlas of the human brain. San Diego: Academic Press, 1997.

25. Watson C, Jack CR, Cendes F. Volumetric magnetic resonance imaging: clinical applications and contributions to the understanding of temporal lobe epilepsy. Arch Neurol 1997;54: 1521-1531.

26. Dunn G, Everitt B. Clinical biostatistics: an introduction to evidence based medicine. Edinburgh: Arnold, 1995.

27. Laakso M, Partanen K, Riekkinen P, et al. Hippocampal volumes in Alzheimer's disease, Parkinson's disease with and without dementia, and in vascular dementia. Neurology 1996; 46:678-681.

28. Warrington EK. Recognition memory test. Windsor: NFERNelson, 1984

29. Wechsler DA. Wechsler Memory Scale, Revised. San Antonio, TX: Psychological Corporation, 1987.

30. Howard D, Patterson K. Pyramids and palm trees: a test of semantic access from pictures and words. Bury St. Edmonds: Thames Valley Test Company, 1992.

31. Warrington E, James M. The visual object and space perception battery. Bury St Edmunds: Thames Valley Test Company, 1991.

32. Braak H, Braak E. Neuropathological staging of Alzheimerrelated changes. Acta Neuropathol 1991;82:239-259.

33. Van-Hoesen GW, Hyman BT, Damasio AR. Entorhinal cortex pathology in Alzheimer's disease. Hippocampus 1991;1:1-8.

34. Rossor MN, Revesz T, Lantos PL, Warrington EK. Semantic dementia with ubiquitin-positive tau-negative inclusion bodies. Brain 2000;123:267-276.

35. Graff-Radford NR, Damasio AR, Hyman BT, et al. Progressive aphasia in a patient with Pick's disease: a neuropsychological, radiologic, and anatomical study. Neurology 1990;40:620-626.

36. Harasty JA, Halliday GM, Code C, Brooks WS. Quantification of cortical atrophy in a case of progressive fluent aphasia. Brain 1996;119:181-190.

37. Lawrence AD, Sahakian BJ. The cognitive psychopharmacology of Alzheimer's disease: focus on cholinergic systems. Neurochem Res 1998;23:787-794.

38. Dolan RJ, Fletcher PC. Dissociating prefrontal and hippocampal function in episodic memory encoding. Nature 1997;388: $582-585$.

39. Shallice T, Fletcher P, Frith CD, Grasby P, Frackowiak RSJ, Dolan RJ. Brain regions associated with acquisition and retrieval of verbal episodic memory. Nature 1994;368: $633-635$.

40. Johnson JK, Head E, Kim R, Starr A, Cotman CW. Clinical and pathological evidence for a frontal variant of Alzheimer's disease. Arch Neurol 1999;56:1233-1239.

41. Minoshima S, Foster NL, Kuhl DE. Posterior cingulate cortex in Alzheimer's disease. Lancet 1994;344:895

42. Aggleton JP, Shaw C. Amnesia and recognition memory: a reanalysis of psychometric data. Neuropsychologia 1996;34:51-62.

43. Wiggs CL, Weisberg J, Martin A. Neural correlates of semantic and episodic memory retrieval. Neuropsychologia 1999;37: $103-118$.

44. Vandenberghe R, Price C, Wise R, Josephs O, Frackowiak RSJ. Functional anatomy of a common semantic system for words and pictures. Nature 1996;383:254-256.

45. Murray EA, Bussey TJ. Perceptual-mnemonic functions of the perirhinal cortex. Trends Cogn Sci 1999;3:142-151.

46. Folstein MF, Folstein SE, McHugh PR. "Mini-mental state": a practical method for grading the cognitive state of patients for the clinician. J Psychiatr Res 1975;12:189-198. 


\section{Differing patterns of temporal atrophy in Alzheimer's disease and semantic dementia}

C. J. Galton, K. Patterson, K. Graham, M.A. Lambon-Ralph, G. Williams, N. Antoun,

B.J. Sahakian and J.R. Hodges

Neurology 2001;57;216-225

\section{This information is current as of March 31, 2008}

$\begin{array}{ll}\begin{array}{l}\text { Updated Information } \\ \text { \& Services }\end{array} & \begin{array}{l}\text { including high-resolution figures, can be found at: } \\ \text { http://www.neurology.org/cgi/content/full/57/2/216 }\end{array} \\ \text { Supplementary Material } & \begin{array}{l}\text { Supplementary material can be found at: } \\ \text { http://www.neurology.org/cgi/content/full/57/2/216/DC1 }\end{array} \\ \text { Permissions \& Licensing } & \begin{array}{l}\text { Information about reproducing this article in parts (figures, tables) } \\ \text { or in its entirety can be found online at: } \\ \text { http://www.neurology.org/misc/Permissions.shtml }\end{array} \\ & \begin{array}{l}\text { Information about ordering reprints can be found online: } \\ \text { http://www.neurology.org/misc/reprints.shtml }\end{array}\end{array}$

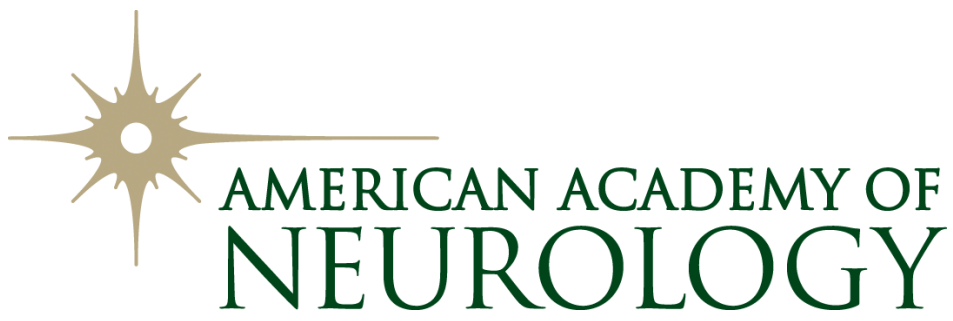

